\title{
Performance and Robustness of Automatic Fluoroscopic Image Calibration in a New Computer Assisted Surgery System
}

\author{
Peter M. Tate, Vladimir Lachine, Liqun Fu, Haniel Croitoru, Marwan Sati \\ Surgical Navigation Specialists, 6509 Airport Rd, Mississauga ON, Canada L4V 1S7 \\ ptate@surgnav.com
}

\begin{abstract}
In order to improve the clinical usefulness of computer-assisted fluoroscopic navigation, a new algorithm to automatically determine the calibration of fluoroscopic images has been developed. This is a challenging task since the intraoperative images acquired from fluoroscopic systems are often poor, making detection of the calibration grid difficult. Several featurebased methods have been implemented to perform bead detection for automatic detection of the calibration grids. The algorithms include support for multiple fields of view, a feature not supported on any computer assisted systems to date. In order to evaluate the performance of the algorithms, special phantoms were made and a cadaver study was performed to challenge the algorithms. One hundred images were acquired using three different C-Arms (OEC 9600, OEC 9800 and Philips BV-300+) using two different fields of view (nine and twelve inch). The chosen method successfully registered the images in ninety-six of the cases. The images that were not successfully registered were of limited clinical value anyway due to the very poor image quality.
\end{abstract}

\section{Introduction}

Computer-assisted fluoroscopic navigation has received strong interest as a new tool for spinal and orthopedic procedures [ 3 - 5 - 6 [7] Computer-assisted fluoroscopic navigation is a technique whereby a conventional C-Arm is equipped with an optically-tracked calibration device that consists of two fiducial grids and a collar that mounts to the image intensifier. When an image is acquired during surgery, the position of the C-Arm is captured and the image is transferred to the computer. After uploading the image onto the C-Arm, the computer detects the fiducial markers and calculates the calibration parameters (image distortions, image-patient transformation) and then superimposes instruments on multi-planar images for navigation. To improve image display, the grids are removed from the image, and the geometric image distortions are removed (the image is "unwarped"). The advantages over conventional fluoroscopy includes reduced radiation exposure, real-time guidance, no need to have the cumbersome image intensifier in the working area during the intervention, and navigation that is available in multiple planes.

Many current systems are challenged by the need to keep the C-Arm console settings constant throughout the surgical procedure (i.e. constant flip, rotation, and 
field of view) before uploading the image. This can disturb the standard clinical workflow since image manipulation is often performed on the console to help the surgeon's orientation with the anatomy or to change the field of view for the area of interest. We have developed an asymmetric grid and an automatic grid detection technique that allows the user to use the C-Arm in a conventional fashion. This paper investigates the performance and robustness of this new technique.

Current systems miss fiducial markers in certain images and it is another objective of this work to improve fiducial detection rate through improved image processing algorithms. Fiducial beads with diameter larger than $3 \mathrm{~mm}$, used in some systems, are not desired since they noticeably obstruct the image, so another objective was to distinguish between 2 and $3 \mathrm{~mm}$ beads arranged in an asymmetric grid for automatic detection of flip, rotate and field of view.

\section{Materials and Methods}

Three C-Arms (OEC 9600, OEC 9800 and Philips BV-300+) were used to acquire fluoroscopic images using the new fluoroscopic navigation system. The grids contained an asymmetric bead pattern that was used to identify flip, rotation and changes in the field of view in the image.

Spinal, hip, and femur phantoms, as well as images from three human cadaver trials were used in this study. The cadavers were specially ordered "large" specimens that provided worst-case quality images (due to the significant signal attenuation) and allowed us to test the robustness of the algorithm. Metallic surgical instruments and implants were placed in both the phantom images and the cadaver images to simulate worst-case obstruction of fiducial markers. One hundred images were analyzed in all.

Results were visually checked to determine accurate bead detection results, and were analyzed with respect to number of false positives, localization accuracy, number of beads detected per image. Ideally we would like to detect all the beads, however because of occlusions and image noise, this is not always possible.

The crucial step in automatic registration is robust bead detection. To perform bead detection, the beads were first segmented from the image by taking the difference between the original image and an image of the background intensity. The difference image was then thresholded using an adaptive method based on the intensity of the background image. This gave us a series of candidate bead positions, the orientation of the grid could then be determined using the Hough transform [4] parameterized using the normal representation of a line. Since the grids are laid out in rows and columns, inputting the candidate positions creates a pattern with strong responses when the rotation angle matches the orientation along the rows and columns, and along the diagonals of the grid. The proper orientation can be determined based on the spacing at that orientation since the spacing along the diagonal will be less than the spacing along the row/column direction. The translation and scale of the grid could be determined by taking projections along the rows and columns of the grids. Since the grid is symmetric, this determined the rotation to within integral intervals of 90 degrees. To resolve flip, the calibration grid was designed to have large beads 
placed along two rows and one column of the proximal plate (note the bead pattern in the first image of Figure 2).

After the initial grid orientation was determined, the rows and columns that may contain large beads were searched based on bead size to determine the final grid orientation. Searching is performed by calculating the average bead sizes of the possible rows and columns that may contain large beads and testing the candidates against each of the eight possible combinations by flipping and rotating the known bead pattern. The best fit is the pattern that yields the largest average size. The most challenging aspect of this process is to determine an appropriate size measure to differentiate the beads. We used the number of pixels in the segmented beads from the first step. Global thresholding provided inconsistent results, therefore an adaptive thresholding based on the background intensity was used which provided a more robust size metric, as well as better bead detection.

Two different algorithms were developed to find the background image, one is a grayscale morphological method based on Top Hat transformatior[4], and the second is based on median filtering.

An example of bead detection can be seen in Figure 1. A special mode is shown where the system displays detected beads with crosses and large beads with circles. The standard mode does not show the bead location with crosses, but rather warns the system of a potential invalid registration.

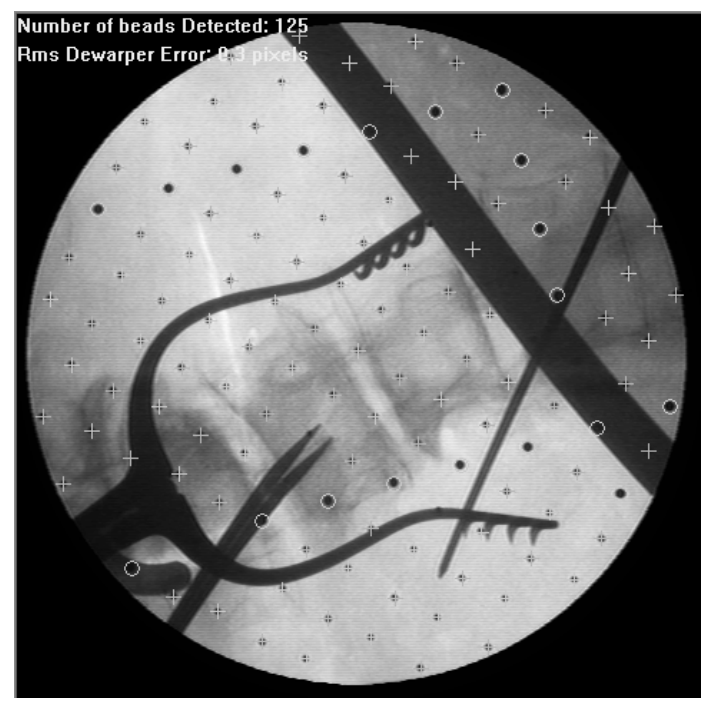

Fig. 1. Successful calibration. A special option allows the user to visualize the bead positions.

Validation of the bead detection was performed both automatically and visually. The automatic validation method was developed for intraoperative use to warn the surgeon of images that may not be properly registered. Automatic validation included checking for a minimum number of beads detected in each of the calibration grids, a check on the distribution of beads in the image, and a check on the magnitude of the distortion recovered from the image based on the unwarping function. The threshold 
for the minimum number of beads varied depending on the field of view of the image. We have found that the magnitude of the distortion in the image is bounded, so if the amount of distortion (measured as the difference between a bead's detected location, and its predicted "ideal" location after dewarping) for any bead is beyond the expected range, the calibration of that image was rejected. If the bead detection fails to meet these specified criteria (which varied depending on the field of view), the image calibration is rejected and a warning is displayed to the user.

After calibration, the beads are removed from the image to improve the surgeons view of the anatomy. Beads are removed by clipping the bead from the image, and performing surface fitting using a high order polynomial. The surgeon is also provided an option to not remove the beads.

\section{Results}

Results were evaluated in terms of the number of successful registrations, the average number of beads detected, the number of falsely detected beads, and the RMS dewarper error. The total number of beads that could be detected in the image varied with the field of view (241 for the twelve-inch field of view, and 137 for the nineinch). The RMS dewarper error was found by calculating the difference between the detected location, and the predicted location found by the unwarping. In addition, each image was visually checked for false detections and to verify that the proper flip, shift, and rotation were determined.

Results from the study are shown in Table 1 . The median method was successful on 96 out of 100 images compared with the morphological method's 92 out of 100 . In each unsuccessful case the failed calibration was automatically found, thus ensuring that a failed image would not be navigated upon. On average the median method also found 3 more beads had a slightly improved RMS dewarper error.

Table 1. Results of automatic registrations.

\begin{tabular}{lllll}
\hline Method & $\begin{array}{l}\text { Number of } \\
\text { Successful } \\
\text { Calibrations }\end{array}$ & $\begin{array}{l}\text { Number of } \\
\text { falsely } \\
\text { detected } \\
\text { beads (Total) }\end{array}$ & $\begin{array}{l}\text { Average } \\
\text { number of } \\
\text { beads } \\
\text { detected }\end{array}$ & $\begin{array}{l}\text { Average RMS } \\
\text { dewarper } \\
\text { error (mm) }\end{array}$ \\
\hline Morphological & 92 & 1 & 186 & 0.43 \\
\hline Median & 96 & 1 & 189 & 0.41 \\
\hline
\end{tabular}

A sample result from bead removal is shown in Figure 3. Bead removal generally performs very well, removing the grid from most parts of the image. Bead detection works well on edges, however bead removal on edges remains a challenge. The current bead removal may cause local blurring because the current surface matching interpolation method may be adversely affected by image content, showing smoothly varying "smudges" where the image contrast changes sharply. 

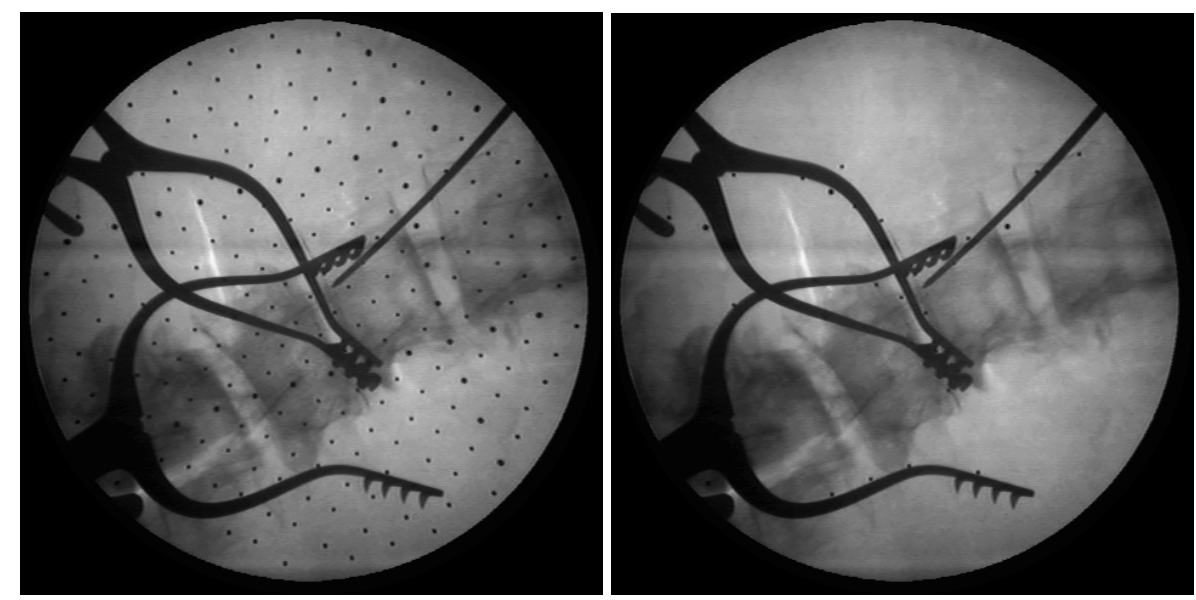

Fig. 2. Challenging image with beads removed. Note that some beads may be detected even if they are not removed.

\section{Discussion}

Of the two bead detection methods, results from median filtering are better than the morphological filtering, though marginally so (see results in Table 1). This is due to the morphological filtering increasing the average gray level of the image, hence the difference between the original and background images is reduced making thresholding more difficult.

Of the images that the bead detection failed, several were high-energy images and did not contain clinically interesting properties. In general, it is expected that some images cannot be automatically registered. This may be caused by a surgical tool in the field of view that obstructs one or more rows of large beads, making the final solution indeterminate, however this should be quite rare in practice. In these cases, users have the option to perform a manual calibration of the image, by setting the proper flip, rotation, and shift.

The system generally performed very well, and was able to find beads near edges and even through some metal instruments! (Figure 3) The performance of many other methods, especially signal-based methods such as cross-correlation, tend to suffer near edges.

A piece of electronics in a patient tracker that was obscuring a number of beads was falsely detected as a bead in one of the images, as is shown in Table 1. Because there were a sufficient number of beads detected elsewhere in the image, this did not affect the overall result.

The feature based technique proved extremely robust at determining the proper orientation of the grid, calculating the proper calibration in all cases where a sufficient number of beads were found. The feature-based method requires only that the beads in the image be aligned in a rectilinear grid, and that the center of the grid be close to 
the center of the image. The actual number of rows and columns in the grid is not important for determining the orientation or spacing.
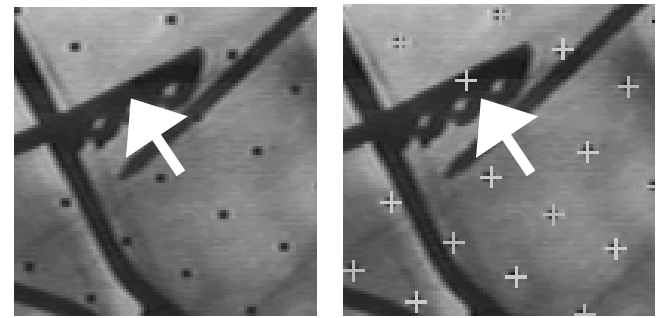

Fig. 3. Left hand image shows beads in the image, right hand image shows crosses representing the detected beads. Note the bead detected through the metal instrument.

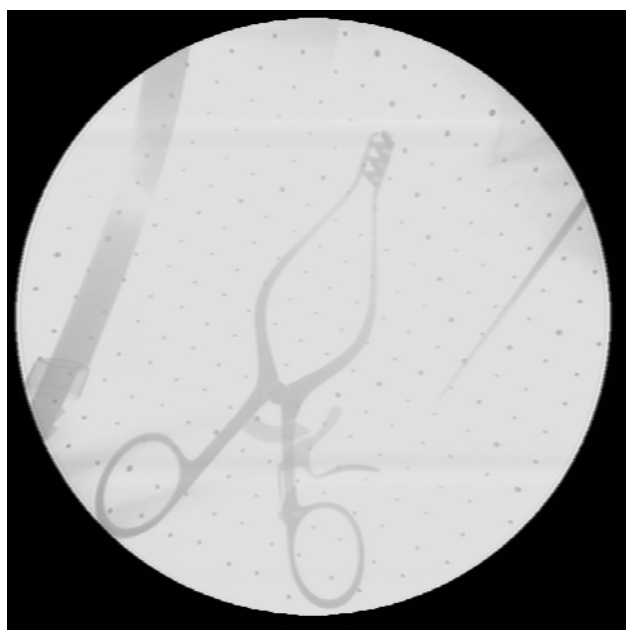

Fig. 4. Example of an unsuccessful calibration because of high-energy fluoroscopy. Note that there is no anatomy visible so the image would not be used clinically.

The bead removal generally performed well, with beads removed near sharp edges causing some blurring. The current surface-fitting algorithm assumes smoothly varying intensity across the image. While this assumption is valid for most parts of the image, surgical tools and implants are characterized by sharp edges. By better modeling the intensity variation in the image, we anticipate that we can deliver better results. In order to minimize the visual artifacts caused by bead removal, the RMS error of the surface fitting is calculated, and if it is deemed too high, as often occurs near the edges of metal instruments, the bead is not removed from the field of view.

\section{Conclusions}

This paper shows the performance and robustness of a new system for intra-operative fluoroscopic image registration. 
- A very large percentage of fiducial beads were detected even under worst-case conditions of large human cadavers with simulated implants and surgical instruments within the field of view. It was even possible in some cases to detect beads that were normally hidden behind metal.

- The system was robust in distinguishing between 2 and $3 \mathrm{~mm}$ beads and automatically detecting image manipulations at the $\mathrm{C}$-arm console such as changing fields of view, image rotations and image flips.

- Bead removal is very satisfactory, however there is room for improvement for the grayscale interpolation to reduce blurring.

\section{References}

[1] Brack C, Burgkart R, Czopf A, Götte H, Roth M, Radig B, Schweikard A (1998) Accurate X-ray-based Navigation in Computer-Assisted Orthopaedic Surgery. Proc CAR Symposium 1998:716-722.

[2] Brack C, Götte H, Gossé F, Moctezuma J, Roth M, Schweikard A (1996) Towards Accurate X-Ray-Camera Calibration in Computer-Assisted Robotic Surgery. Proc CAR Symposium 1996:721-728.

[3] Foley, Kevin T., MD, Simon, David A, PhD, Rampersaud, Y. Raja MD (2000) Virtual Fluoroscopy. Operative Techniques in Orthopaedics 10(1) pp77-81

[4] Gonzalez, Rafael C., Woods, Richard E. (1992) Digital Image Processing - Third Edition. Addison-Wesley Publishing Company.

[5] Hofstetter R., Slomczykowski M., Sati M. and Nolte L.-P. "Fluoroscopy as an imaging means for computer assisted surgical navigation", 1999, Computer Aided Surgery 4:65-76.

[6] Hofstetter R., Slomczykowski M., Krettek C., Sati M. and Nolte L.-P. "Computer Assisted Fluoroscopy Based Reduction of Femoral Fractures and Anteversion Correction", 2000, Computer Aided Surgery 5:311-325

[7] Joskowicz L, Taylor RH, Williamson B, Kane R, Kalvin A, Guéziec A, Taubin G, Funda J, Gomory S, Brown L, McCarthy J, Turner R (1995) Computer Integrated Revision Total Hip Replacement Surgery: Preliminary Report. Proc $2^{\text {nd }}$ MRCAS Symposium, 193-202.

[8] Viant WJ, Phillips R, Griffiths JG, Ozanian TO, Mohsen AMMA, Cain TJ, Karpinski MRK, Sherman KP (1997) A computer assisted orthopaedic surgical system for distal locking of intramedullary nails. Proc Instn Mech Engrs 211 (H):293-299. 